\title{
Hyperglycaemia attenuates in vivo reprogramming of pancreatic exocrine cells to beta cells in mice
}

\author{
Claudia Cavelti-Weder ${ }^{1} \cdot$ Weida $\mathrm{Li}^{2,3} \cdot$ Adrian Zumsteg $^{3}$ • \\ Marianne Stemann-Andersen ${ }^{1}$ • Yuemei Zhang ${ }^{3} \cdot$ Takatsugu Yamada $^{1} \cdot$ Max Wang $^{3}$. \\ Jiaqi Lu ${ }^{3}$ • Agnes Jermendy ${ }^{1}$ • Yong Mong Bee ${ }^{1} \cdot$ Susan Bonner-Weir $^{1}$ • \\ Gordon C. Weir ${ }^{1}$ • Qiao Zhou ${ }^{3}$
}

Received: 7 August 2015 / Accepted: 17 November 2015 / Published online: 23 December 2015

(C) Springer-Verlag Berlin Heidelberg 2015

\begin{abstract}
Aims/hypothesis Reprogramming of pancreatic exocrine to insulin-producing cells by viral delivery of the genes encoding transcription factors neurogenin-3 (Ngn3), pancreas/duodenum homeobox protein $1(P d x I)$ and MafA is an efficient method for reversing diabetes in murine models. The variables that modulate reprogramming success are currently ill-defined.

Methods Here, we assess the impact of glycaemia on in vivo reprogramming in a mouse model of streptozotocin-induced beta cell ablation, using subsequent islet transplantation or insulin pellet implantation for creation of groups with differing levels of glycaemia before viral delivery of transcription factors.

Results We observed that hyperglycaemia significantly impaired reprogramming of exocrine to insulin-producing cells in their quantity, differentiation status and function. With hyperglycaemia, the reprogramming of acinar towards beta cells was less complete. Moreover, inflammatory tissue
\end{abstract}

Claudia Cavelti-Weder and Weida Li contributed equally to this study

Electronic supplementary material The online version of this article (doi:10.1007/s00125-015-3838-7) contains peer-reviewed but unedited supplementary material, which is available to authorised users.

Qiao Zhou

qiao_zhou@harvard.edu

1 Section on Islet Cell and Regenerative Biology, Joslin Diabetes Center, Harvard University, Boston, MA, USA

2 Translational Medical Center for Stem Cell Therapy and Institute for Regenerative Medicine, Shanghai East Hospital, Tongji University School of Life Sciences and Technology, Shanghai, The People's Republic of China

3 Department of Stem Cell and Regenerative Biology, Harvard University, Sherman Fairchild 258C, 7 Divinity Ave, Cambridge, MA 02138, USA changes within the exocrine pancreas including macrophage accumulation were found, which may represent the tissue's response to clear the pancreas from insufficiently reprogrammed cells.

Conclusions/interpretation Our findings shed light on normoglycaemia as a prerequisite for optimal reprogramming success in a diabetes model, which might be important in other tissue engineering approaches and disease models, potentially facilitating their translational applications.

Keywords Diabetes - Exocrine to beta cell reprogramming · Hyperglycaemia

$\begin{array}{ll}\text { Abbreviations } \\ \text { ChgA } & \text { Chromogranin A } \\ \text { DT } & \text { Diphtheria toxin } \\ \text { DTR } & \text { Diphtheria toxin receptor } \\ \text { FDR } & \text { False discovery rate } \\ \text { HSP60 } & \text { Heat shock protein } 60 \\ \text { Hyper } & \text { Hyperglycaemic group } \\ \text { InsP } & \text { Insulin pellets } \\ \text { IPS cell } & \text { Induced pluripotent stem cell } \\ \text { KEGG } & \text { Kyoto Encyclopedia of Genes and Genomes } \\ \text { Normo } & \text { Normoglycaemic group } \\ \text { Nx } & \text { Nephrectomy } \\ \text { panCK } & \text { Pan-cytokeratin } \\ \text { PDL } & \text { Partial duct ligation } \\ \text { PTF1A } & \text { Pancreas specific transcription factor } 1 \mathrm{a} \\ \text { Rag } & \text { B- } \\ \text { STZ } & \text { Streptozotocin } \\ \text { Tx } & \text { Transplantation } \\ \text { WT } & \text { Wild type }\end{array}$




\section{Introduction}

For type 1 and type 2 diabetes, there is great interest in the generation of new beta cells for replacement therapy. Four approaches have received considerable attention: replication of existing beta cells [1-3]; neogenesis of new beta cells from precursor cells [4-7]; embryonic or induced pluripotent stem (iPS) cell differentiation into beta cells $[8,9]$; and direct reprogramming of non-beta to beta cells [10-17]. For the last approach, varying success has been achieved in vivo by administration of transcription factors [12-14, 17] or cytokines [10], or the ablation of forkhead box protein $\mathrm{O} 1$ (FoxO1) in enteroendocrine progenitor gut cells [18]; furthermore, evidence exists for the conversion of alpha or delta cells to beta cells after near complete beta cell loss $[11,16]$. Reprogramming of acinar to beta cells has been shown to yield insulin ${ }^{+}$cells that can persist and function for at least 13 months [14], which raises questions about potential clinical applications.

Among the many environmental factors that could influence reprogramming, glucose is of particular interest because it plays a dominant role in the maintenance of beta cell mass [19]. Beta cell hyperplasia has been linked to glucose metabolism in studies using genetic tools to reduce glucokinase activity [20,21]. However, chronic hyperglycaemia can exert harmful effects on beta cells leading to a marked loss of glucose-stimulated insulin secretion [22-27] and changes in gene expression consistent with dedifferentiation or apoptosis $[15,28,29]$. However, nothing is known about the effect of hyperglycaemia on in vivo acinar to beta cell reprogramming.

In the present study, we asked whether and how glucose influences in vivo reprogramming of exocrine to insulin ${ }^{+}$cells using the three transcription factors neurogenin-3 (NGN3), pancreas/duodenum homeobox protein 1 (PDX1), and MafA. Streptozotocin (STZ)-treated mice were subjected to either islet transplantation or insulin pellet (InsP) implantation to create distinct glycaemic groups before viral delivery of transcription factors [30]. We found that overt hyperglycaemia negatively impacts reprogramming of exocrine to insulin ${ }^{+}$ cells in terms of their quantity, differentiation status and function. This reprogramming was accompanied by an inflammatory response within the exocrine pancreas with macrophage accumulation. Thus, we found that reprogramming success depends on not only the delivery of transcription factors but also a normoglycaemic environment.

\section{Methods}

Animals Male 6-week-old B6.129S7-Rag $1^{\text {tm1Mom }} / \mathrm{J}$ mice $\left(\mathrm{Rag}^{-/-}\right)$were obtained from Jackson Laboratories (Bar Harbor, ME, USA). All procedures were approved by the Animal Care and Use Committees of both the Joslin Diabetes Center and Harvard University.
Polycistronic virus construction $\mathrm{Ngn} 3$ (also known as Neurog3)/Pdx1/MafA with the fluorescence marker Cherry on a polycistronic system, or Cherry alone, were cloned into the $\mathrm{pad} / \mathrm{CMV} / \mathrm{V} 5$ adenoviral vector by the gateway system as previously described [30], and referred to as M3Cherry and Cherry, respectively.

Administration of STZ A single dose of STZ (Sigma, St Louis, MO, USA), $180 \mathrm{mg} / \mathrm{kg}$ freshly dissolved $(20 \mathrm{mg} / \mathrm{ml})$ in $1.093 \mathrm{mmol} / \mathrm{l}$ citrate buffer ( $\mathrm{pH} 4.5)$, was injected intraperitoneally into $\mathrm{Rag}^{-/-}$mice after an $8 \mathrm{~h}$ daytime fast.

Islet isolation and transplantation, InsP implantation and blood glucose measurements After STZ-treatment, groups with differing levels of glycaemia were created using the following protocols. In the hyperglycaemic group, diabetic mice received two insulin pellets (LinShin Canada, Toronto, ON, Canada) by subcutaneous implantation 3-8 days after the STZ-injection. These InsP were sub-therapeutic allowing hyperglycaemia with preservation of reasonable health; additional InsP were implanted upon 10\%-20\% weight loss. In the normoglycaemic group, islet transplantation (Tx) was employed to achieve normoglycaemia. Islets from adult 6-week-old male C57BL/6J mice were isolated by collagenase digestion [31] with rodent Liberase RI (Roche, Indianapolis, IN, USA) and purified by gradient separation using Histopaque-1077 (Sigma). Three to eight days after STZ, 6-week-old $\mathrm{Rag}^{-/-}$mice were transplanted with 500 islets under the right kidney capsule [32]. Blood glucose levels were measured on blood from snipped tails with a glucometer (One touch Ultra, Life Scan, Milpitas, CA, USA).

Virus injection Two weeks after the islet Tx or InsP implantation, the pancreatic tail was injected with $100 \mu \mathrm{l}$ of purified virus at one-two loci (titre $2 \times 10^{10}$ plaque-forming units $/ \mathrm{ml}$ ) with a 3/10 ml Insulin Syringe (Becton Dickinson, Franklin Lakes, NJ, USA) as previously described [30].

Nephrectomy In a subset of mice, the right kidney containing the islet graft was removed 10 or 25 days after virus injection. Thereafter, glucose control fully depended on induced insu$\operatorname{lin}^{+}$cells in the pancreas.

Tissue collection and immunostaining Adult mouse pancreases were fixed with $4 \%$ paraformaldehyde for $2 \mathrm{~h}$ at $4^{\circ} \mathrm{C}$. Samples were subsequently incubated in $30 \%$ sucrose solution overnight $(6-12 \mathrm{~h})$ and embedded with O.C.T. Compound (Tissue-Tek, Torrance, CA, USA). The antibodies used for immunohistochemistry are listed in electronic supplementary material (ESM) Table 1. DAPI was used for nuclear staining. Confocal images of pancreatic sections were taken on a Zeiss LSM710 (Zeiss, Thornwood, NY, USA). 
Quantification of insulin-containing cells To estimate the number of reprogrammed cells, insulin ${ }^{+}$cells in every fifth section of the entire infected pancreatic portion were counted by hand and multiplied by the factor 5 using a confocal microscope (Zeiss LSM 710 [Zeiss] at Joslin or a Nikon Eclipse Ti-S [Nikon, Melville, NY, USA] microscope in Cambridge). For quantifications of ratios (e.g. heat shock protein 60 $[\text { HSP60 }]^{+}$:Cherry ${ }^{+}$cells), we counted cells in four-five independent slides from the infected region of each animal.

Gene profile analysis On day 10 after reprogramming, pancreases were dissociated by perfusion via the bile duct of a Liberase-containing solution $(25 \mathrm{mg} / \mathrm{ml})$. Endogenous islets were separated from the exocrine fraction by gradient centrifugation and dithizone staining. The infected exocrine cell fraction was furthered dissociated into single cells by Liberase, dispase and elastase. Cherry ${ }^{+}$cells were collected by FACS with FACSaria (BD Bioscience, San Jose, CA, USA). RNA was extracted from the Cherry ${ }^{+}$cells. Gene profiling by Illumina microarrays (San Diego, CA, USA) was analysed to assess differential gene expression of cells reprogrammed in hyperglycaemia and normoglycaemia. We used gene set enrichment analysis as well as the Kyoto Encyclopedia of Genes and Genomes (KEGG) pathway database to find significantly enriched gene sets as defined by false discovery rate (FDR) $<25 \%$ to account for multiple testing.

\section{Bone marrow Tx of CD11b-DTR or wild-type donors and} diphtheria toxin injection Six-week-old male $\mathrm{Rag}^{-/-}$mice were lethally irradiated using 9 Gy $(2 \times 4.5$ Gy $4 \mathrm{~h}$ apart). After $6 \mathrm{~h}$, mice were transplanted by intravenous injection of $10^{7}$ erythrocyte-depleted (ACK lysis buffer) bone marrow cells extracted from the femur and tibia of 8-week-old $\mathrm{Rag}^{-1-}$ or $\mathrm{Rag}^{-1-}$;CD11b-diphtheria toxin receptor (DTR) transgenic mice. Rag $^{-1-}$;CD11b-DTR mice are B6.FVB-Tg(ITGAMDTR/EGFP)34Lan/J (Jackson Laboratories) crossed for five generations into $\mathrm{Rag}^{-/-}$mice. Four weeks after bone marrow transplantation, adenovirus injection was performed as above. For macrophage depletion, diphtheria toxin (DT; Sigma) was administered intraperitoneally to all mice at $25 \mathrm{ng} / \mathrm{g}$ body weight (in TRIS-buffered saline $\mathrm{pH}$ 7.6) on days $0,2,4,6,8$ and 10 after virus injection. PCR was performed on crude infected pancreas (see ESM Table 2 for primers).

Data analysis All animals have been included in data analysis. Data are expressed as mean \pm SEM unless otherwise indicated. Mann-Whitney or $t$ tests were used as indicated to estimate statistical significance. Correlations were calculated with the Spearman's $r$ test. Contingency tables were analysed by $\chi^{2}$ test. A $p$ value $<0.05$ was considered statistically significant.

\section{Results}

Reprogramming with hyperglycaemia leads to fewer insulin ${ }^{+}$ cells To determine if hyperglycaemia has a negative influence on reprogramming, STZ-treated diabetic $\mathrm{Rag}^{-/-}$mice were randomly assigned to a normoglycaemic group by transplantation of 500 islets of C57-mice or a hyperglycaemic group by implantation of InsP to keep animals viable yet hyperglycaemic. M3Cherry or Cherry control viral constructs were injected into the pancreas 2 weeks later, and animals were killed after 10 or 25 days (Fig. 1a). Mice treated with InsP stayed hyperglycaemic $(30.5 \pm 0.4 \mathrm{mmol} / \mathrm{l}, n=18)$, while transplanted animals were normoglycaemic $(9.2 \pm 0.3 \mathrm{mmol} / \mathrm{l}$, $n=27$; Fig. 1b). Regardless of glucose levels, reprogramming with the M3Cherry viral construct led to the appearance of single or clustered insulin ${ }^{+}$cells in the pancreatic tail with minimal numbers of Cherry ${ }^{+}$cells stained for glucagon, somatostatin or pancreatic polypeptide (Fig. 1c, ESM Fig. 1a-c). On day 10 , recipients of islet transplants had $141 \pm 22 \times 10^{3}$ insulin $^{+}$cells, while InsP recipients had $84 \pm 20 \times 10^{3}$ (both $n=5 ; p=0.2$, Fig. 1d, ESM Fig. 2a). The number of reprogrammed insulin ${ }^{+}$cells in normoglycaemic animals increased from day 10 to $25\left(233 \pm 25 \times 10^{3}, n=4\right)$ in contrast to the hyperglycaemic group with an unchanged number of reprogrammed cells $\left(79 \pm 10 \times 10^{3}, n=5\right.$, Fig. $\left.1 \mathrm{~d}\right)$. Thus, the estimated total number of reprogrammed insulin ${ }^{+}$cells differed significantly in hyperglycaemic and normoglycaemic animals on day 25 ( $p<0.05$, Fig. 1d). For individual animals at each time point (day 10 and 25), there was an inverse correlation between mean glucose levels after reprogramming and the number of reprogrammed insulin ${ }^{+}$cells (day 10: $r=-0.75, p=0.003[n=13]$; day 25: $r=-0.94, p=0.02$ $[n=6]$, ESM Fig. 2b,c). This inverse correlation even existed in normoglycaemic mice receiving mouse or rat islets, the latter having lower glucose levels due to rat beta cells having lower glucose set-points for secretion (insert ESM Fig. 2b).

With hyperglycaemia, reprogrammed cells show preserved reprogramming initiation but then less differentiation toward a beta cell phenotype We assessed the temporal sequence of reprogramming events. Cell size reduction has been described as an early morphological response to iPS cell and exocrine to beta cell reprogramming [17, 33]. Moreover, we have previously shown that acinar to beta cell reprogramming follows a temporal sequence with acinar cells adopting first an endocrine and then beta cell fate [34]. Thus, reprogramming events were followed over time: (1) initiation of reprogramming as assessed by cell size reduction; (2) endocrine commitment by chromogranin $\mathrm{A}(\mathrm{ChgA})$ expression; and (3) beta cell commitment by insulin expression.

Maximal diameters of $\mathrm{Cherry}^{+}$cells were determined in M3Cherry-injected animals and Cherry-injected controls. In both hyperglycaemic (hyper) and normoglycaemic (normo) 

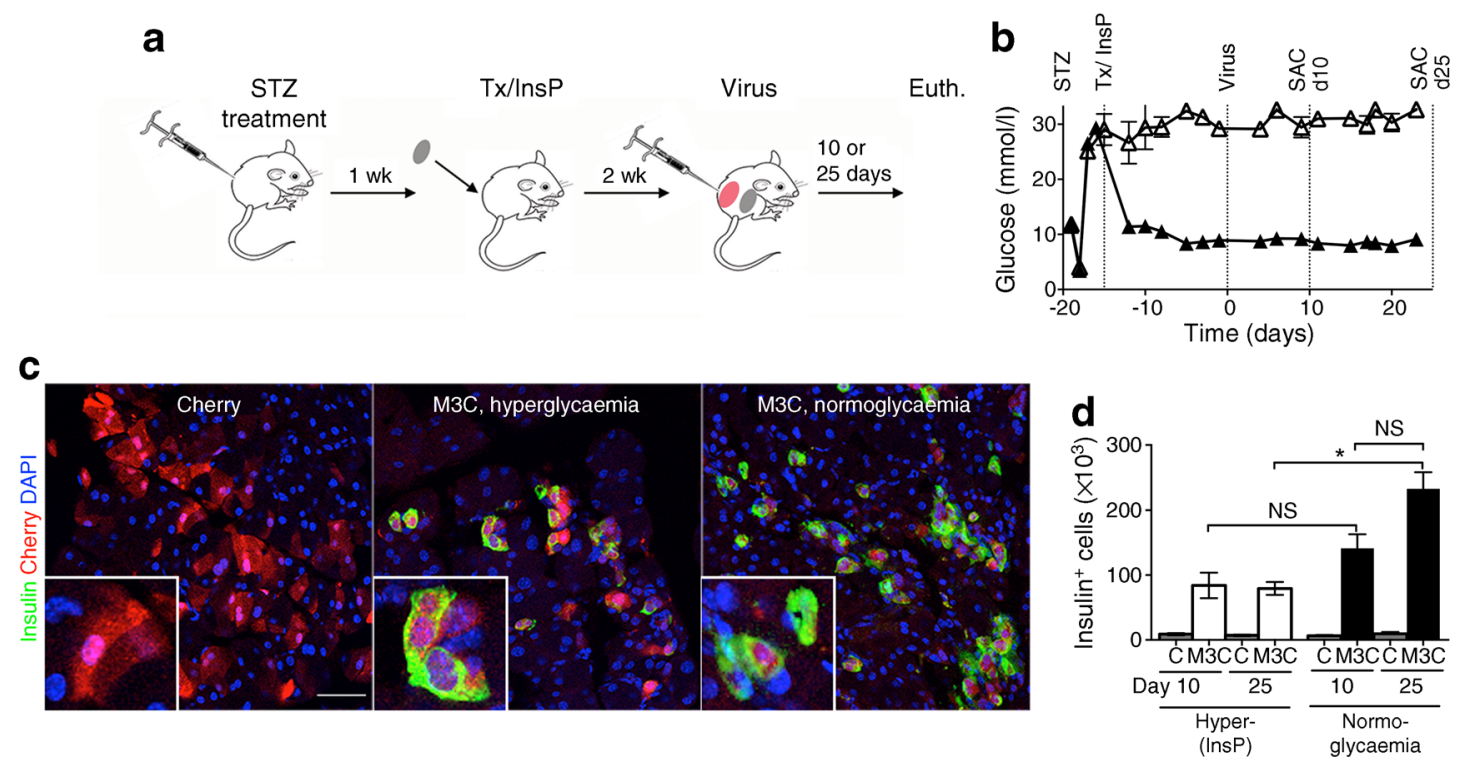

Fig. 1 Reprogramming with hyperglycaemia produces fewer insulin ${ }^{+}$ cells. (a) $\mathrm{Rag}^{-/-}$mice were treated with $180 \mathrm{mg} / \mathrm{kg} \mathrm{STZ}$ and 1 week later subjected to Tx with mouse islets or InsP implantation to create distinct glycaemic groups. Two weeks later, Cherry or M3Cherry viral constructs were injected and animals euthanised (Euth.) 10 or 25 days later for quantification of insulin ${ }^{+}$cells. (b) Blood glucose levels differed significantly between the hyperglycaemic group (open triangles) and normoglycaemic group with islets transplants (closed triangles; $p<0.01$ at all time points). SEMs smaller than symbols are not displayed; d10/d25, day 10/25. (c) M3Cherry injection led to appearance of single or

conditions, cell sizes of Cherry ${ }^{+}$insulin $^{+}$(hyper $18.1 \pm 0.5 \mu \mathrm{m}$, normo $18.3 \pm 0.4 \mu \mathrm{m}$ ) as well as Cherry ${ }^{+}$insulin ${ }^{-}$(hyper 17.5 $\pm 0.6 \mu \mathrm{m}$, normo $18.3 \pm 0.4 \mu \mathrm{m}$ ) cells were significantly smaller in M3Cherry-treated animals compared with Cherry $^{+}$cells in Cherry-injected controls (hyper 27.7 $\pm 0.4 \mu \mathrm{m}$, normo $28.7 \pm 0.2 \mu \mathrm{m}$, all comparisons $p<0.05$, Fig. 2a), indicating preserved reprogramming initiation by cell size reduction, irrespective of glucose levels.

The endocrine commitment as assessed by ChgA expression was $91.2 \pm 2.3 \%$ of Cherry ${ }^{+}$cells in hyperglycaemic mice on day 10 , while virtually all Cherry ${ }^{+}$cells co-expressed ChgA in normoglycaemic animals $(99.0 \pm 0.4 \%, p=0.008$; Fig. 2b), suggesting impaired endocrine commitment with hyperglycaemia. This difference in ChgA expression was also seen on a qualitative level, as ChgA expression in hyperglycaemic animals was much weaker and more inhomogeneous compared with its strong and even expression in normoglycaemia.

Beta cell commitment was assessed by the proportion of infected Cherry ${ }^{+}$cells stained for insulin. On day 10, the percentage of insulin ${ }^{+} /$Cherry $^{+}$cells was significantly lower in hyperglycaemic $(37.2 \pm 4.9 \%, n=5)$ compared with normoglycaemic mice $(57.3 \pm 5.8 \%, n=5$; Fig. $2 \mathrm{c})$. While by day 25 in hyperglycaemia the percentage of insulin ${ }^{+} /$Cherry $^{+}$ cells only increased to $57.0 \pm 4.5 \%(n=5)$, with normoglycaemia clustered insulin ${ }^{+}$cells irrespective of glucose levels (insulin, green; Cherry, red; DAPI, blue; shown day 10, inserts for Cherry ${ }^{+}$and insulin ${ }^{+}$ cells); scale bar, $50 \mu \mathrm{m}$. (d) From day 10 to day 25 the total number of insulin $^{+}$cells increased in normoglycaemic animals (black bars, 141 $\pm 22 \times 10^{3}[n=5]$ day $10 ; 233 \pm 25 \times 10^{3}[n=4]$ day 25), but not hyperglycaemic animals (white bars, $84 \pm 20 \times 10^{3}[n=5]$ day $10 ; 79$ $\pm 10 \times 10^{3}[n=5]$ day 25), leading to a significant difference between the two glycaemic groups by day $25(p=0.016)$.; ${ }^{*} p<0.05$ (Mann-Whitney test). C, Cherry; M3C, M3Cherry

reprogrammed cells exhibited a reprogramming efficiency of $79.0 \pm 6.0 \%$ insulin $^{+} /$Cherry $^{+}$cells $(n=3)$, indicating increasing commitment toward the beta cell lineage (Fig. 2c). This went along with significantly lower expression of NKX2.2 (NK2 homeobox 2) protein in reprogrammed cells of hyperglycaemic animals $(72.2 \pm 5.8 \%, n=3)$ compared with normoglycaemic animals $(87.3 \pm 3.3 \%, n=3, p<0.05, t$ test). Taking these findings together, hyperglycaemia does not affect reprogramming initiation as reprogrammed cells still undergo cell size reduction, yet inhibits the endocrine and beta cell commitment later in this temporal sequence.

Normoglycaemia during reprogramming is required for function of reprogrammed cells When M3Cherry viral constructs were injected into hyperglycaemic animals that had received InsP-implants, sustained normalisation of glucose levels was not achieved (not shown). To determine at what stage hyperglycaemia affects the function of reprogrammed cells, nephrectomies $(\mathrm{Nx})$ of the kidneys carrying the islet grafts were performed early (day 10) or late (day 25) after reprogramming. After Nx, glycaemia fully depended on reprogrammed cells as endogenous beta cells had previously been ablated by STZ (Fig. 3a). Hyperglycaemia defined as glucose $>11.1 \mathrm{mmol} / \mathrm{l}$ in the 2 weeks following Nx occurred in 5/6 animals in the early $\mathrm{Nx}$ group while $1 / 6$ animal was hypoglycaemic as opposed to $2 / 7$ hyperglycaemic, 3/7 normoglycaemic and 2/7 hypoglycaemic 

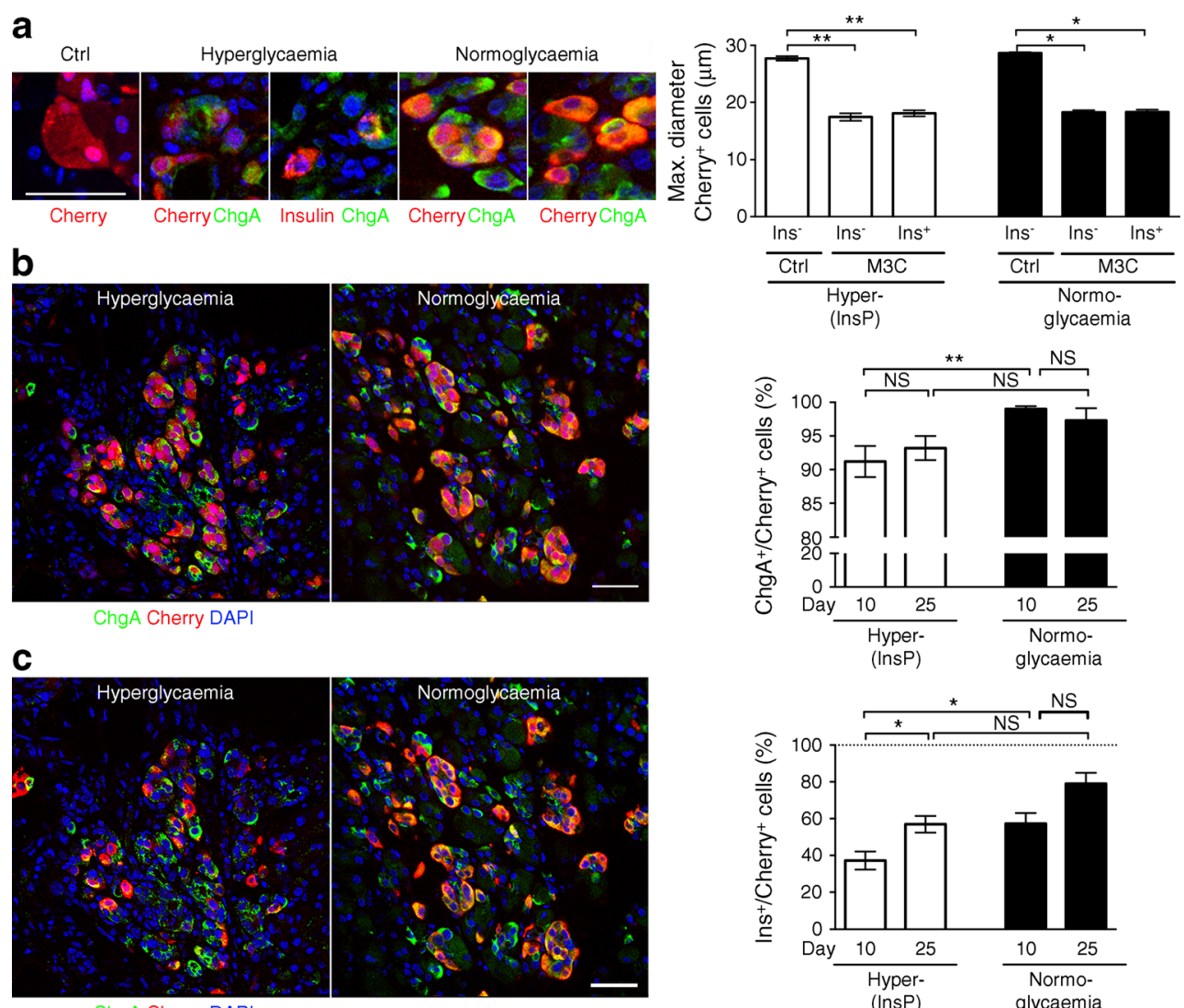

ChgA Cherry DAPI

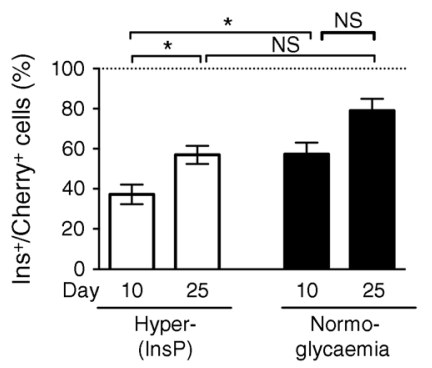

Fig. 2 With hyperglycaemia, reprogrammed cells show preserved initial cell size reduction, but progression to a differentiated phenotype is significantly reduced. See Fig. 1a for set-up of experiments. (a) Cell size: reduction of maximal cell diameters as a measure for reprogramming initiation showed comparable values in hyperglycaemic $(n=6)$ and normoglycaemic $(n=5)$ M3Cherry-injected animals, which were significantly smaller (all $p<0.05$ ) compared with Cherry-injected controls $(n=4)$. (b) Endocrine commitment: on day 10 in hyperglycaemia, reprogrammed Cherry ${ }^{+}$cells expressed significantly less ChgA as a marker of endocrine commitment compared with normoglycaemia (each group $n=5$ ). On day 25 , a new population of $\mathrm{ChgA}^{+}$insulin $^{-}$cells had emerged in the presence of hyperglycaemia $(n=4)$, while most cells reprogrammed in normoglycaemia were Cherry $^{+} \mathrm{ChgA}^{+}$insulin $^{+}(n=3)$. (c) Beta cell commitment: beta cell differentiation as assessed by insulin positivity was less developed $(p=0.03)$ in hyperglycaemia compared with normoglycaemia on day 10 (each group $n=5$ ) and day 25 (normo $n=3$, hyper $n=5, p=0.07$ ). Immunohistochemistry shown from day 10 ; scale bars, $50 \mu \mathrm{m}$; Ctrl, control; M3C, M3Cherry; Max., maximum. $* p<0.05, * * p<0.01$

cells $3.6 \pm 0.5, n=5$ ). Such tubular complexes were not found in normoglycaemic M3Cherry-injected animals at this time point (ratio of $\mathrm{HSP}^{+} 0^{+}$: Cherry ${ }^{+}$cells $1.2 \pm 0.1, n=5$; Fig. 4a, ESM Fig. 3), and by day 25 they were no longer predominant in either group (ratio $\mathrm{HSP}^{+} 0^{+}$Cherry $^{+}$cells $1.0 \pm 0.01$ [hyper, $n=3$ ] and $1.1 \pm 0.05$ [normo, $n=3$ ]; Fig. 4a).

panCK(pan-cytokeratin), a marker of pancreatic duct epithelial cells, was found to be strongly expressed in the tubular complexes of hyperglycaemic M3Cherry-injected animals on day 10 (ratio panCK ${ }^{+}$: Cherry $^{+}$cells $3.9 \pm 0.6$ [hyper, $n=5$ ] vs $0.6 \pm 0.08$ [normo, $n=5]$ ]), while this differential was gone by day 25 (ratio panCK ${ }^{+}:$Cherry $^{+}$cells $1.0 \pm 0.2$ [hyper, $n=4$ ] vs $0.4 \pm 0.02$ [normo, $n=4$ ]; Fig. 4b, ESM Fig. 3). As with HSP60 expression, panCK ${ }^{+}$tubular complexes were not found in hyperglycaemic Cherry-injected controls and noninfected areas of M3Cherry-injected animals (not shown). With normoglycaemia, the majority of reprogrammed cells were found in clusters potentially representing former exocrine acini. 


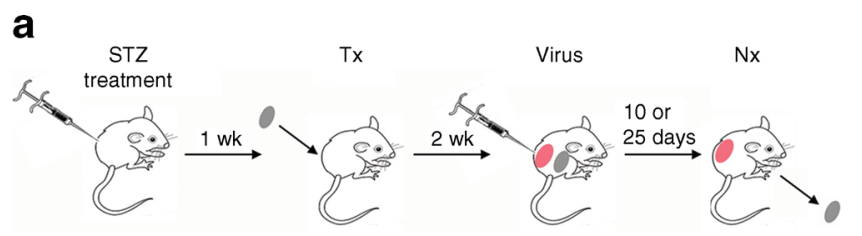

b

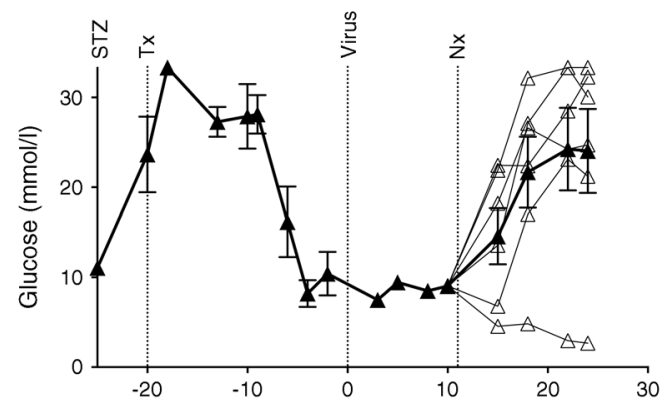

C

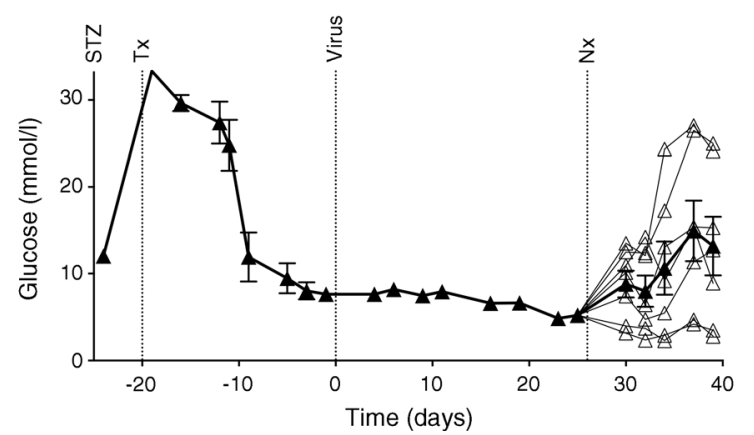

Fig. 3 A minimal time window of normoglycaemia is required to achieve adequate function in reprogrammed cells. (a) $\mathrm{Rag}^{-/-}$mice 6 weeks of age were treated with STZ, transplanted 1 week later with mouse islets, and then 2 weeks later injected with M3Cherry viral construct. Islet cell grafts were removed on day 10 (early Nx) or 25 (late Nx) after virus injection, and blood glucose levels monitored. (b, c) Glucose levels at 1 week after $\mathrm{Nx}(p<0.05)$ and development of hyperglycaemia (5/6 vs $2 / 7$ animals, $\left.\chi^{2} p=0.048\right)$ were significantly different between the early and late Nx groups, respectively. Virus, virus injection

This arrangement was less pronounced with hyperglycaemia, consistent with disrupted pancreatic architecture (Fig. 2c). Together, these findings support the notion that hyperglycaemia prolongs the time of inflammatory exocrine tissue remodelling.

Rapid downregulation of the exocrine gene programme is known to precede adoption of an insulin ${ }^{+}$phenotype [34]. Thus, impaired reprogramming in hyperglycaemia might be due to a failure of acinar cells to efficiently downregulate their exocrine cell programme. Indeed, we found higher expression of PTF1A (pancreas specific transcription factor, 1a) protein (Fig. 4c) in reprogrammed cells of hyperglycaemic $(20.4 \pm 5.3 \%, n=3$ insert $)$ relative to that in normoglycaemic animals by day $10(9.3 \pm 2.8 \%$, $n=3, p<0.05, t$ test). These findings provide additional information about the adverse effects of hyperglycaemia on reprogramming.

Exocrine tissue remodelling is associated with a transient macrophage influx in infected areas Microarray analyses were performed on Cherry ${ }^{+}$cells harvested 7 days after reprogramming. Strikingly, differences in gene expression predominantly concerned inflammatory markers, which were more prominent in the hyperglycaemic condition. By gene set enrichment analysis, we found 25 enriched gene sets in hyperglycaemia defined by an FDR $<25 \%$ to account for multiple testing, 13 of which were directly connected with inflammation or apoptosis (Table 1).

Therefore, we examined the involvement of immune cells in the reprogramming failure of hyperglycaemic animals. As $\mathrm{Rag}^{-/-}$mice do not harbour mature $\mathrm{B}$ and $\mathrm{T}$ lymphocytes, we focused on the role of macrophages. Immunohistochemistry revealed increased numbers of macrophages as assessed by $\mathrm{F} 4 / 80$ staining in areas of reprogramming in hyperglycaemic compared with normoglycaemic M3Cherry-injected animals on day $10\left(\mathrm{~F} 4 / 80^{+}\right.$cells $/ \mathrm{mm}^{2}: 1,501 \pm 263$ [hyper, $\left.n=5\right]$ vs $625 \pm 65$ [normo, $n=4$ ]; Fig. 5a). On day 25, however, the number of macrophages had declined in both glycaemic conditions ( $\mathrm{F} 4 / 80^{+}$cells $/ \mathrm{mm}^{2}: 562 \pm 158$ [hyper, $\left.n=4\right]$ vs $268 \pm 23$ [normo, $n=4$ ]; Fig. 5a), demonstrating the transient nature of the inflammatory response. Macrophages were sparse in hyperglycaemic Cherry-injected controls and in uninfected areas of M3Cherry-injected animals (uninfected areas $\mathrm{F} 4 / 80^{+}$cells $/ \mathrm{mm}^{2}: 69 \pm 15$ [hyper, $n=5$ ] vs $77 \pm 15$ [normo, $n=4$ ] day $10 ; 30 \pm 8$ [hyper, $n=5$ ] vs $30 \pm 5$ [normo, $n=4$ ] day 25). Double-staining for tubular complexes and macrophages revealed their spatial relationship with macrophages adjacent to the tubular structures (ESM Fig. 4).

Macrophages in the infected pancreas clear tissue from partially reprogrammed cells If macrophages were directly deleterious to reprogramming success, reducing their number during or after viral delivery of transcription factors could give rise to more reprogrammed cells. We established a model of macrophage depletion by whole body irradiation, followed by bone marrow Tx of CD11b-DTR or wild-type (WT) donors and DT injections for 10 days to ablate CD11b-DTR cells (Fig. 5b). The 10 day time point was analysed for macrophage infiltration and reprogramming efficiency. As expected, WT transplanted mice had significantly higher macrophage infiltration (macrophages $/ \mathrm{mm}^{2} 640 \pm 120$ [SD], $n=4$ ) compared with CD11b-DTR transplanted mice treated with DT (macrophages $/ \mathrm{mm}^{2} 333 \pm 71$ [SD], $n=4$; Fig. $5 \mathrm{c}$ ). mRNA isolated from infected pancreas confirmed reduced presence of macrophages by lower Emrl (also known as Adgre1) expression (the gene product encoding F4/80 antigen) in CD11b-DTR transplanted mice, whereas adenoviral M3Cherry was expressed at similar levels (Fig. $5 \mathrm{c}$ ). We also found strong mRNA downregulation ( $>20$-fold) of the M2-macrophage polarisation marker Retnla (encoding resistin like alpha) in CD11b-DTR transplanted mice, concomitant with a sevenfold upregulation of Tnf $\alpha$ in the same tissue (ESM Fig 5). 


\section{a}

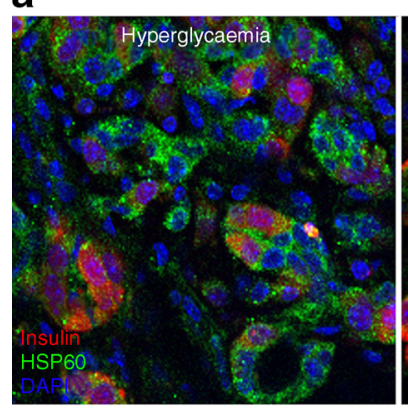

b

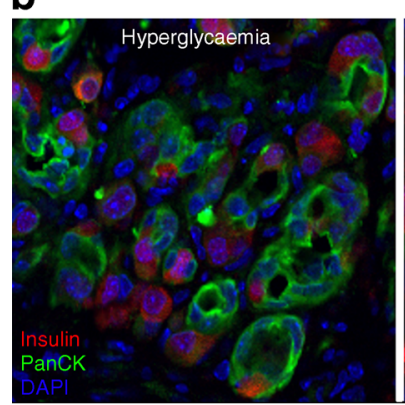

c
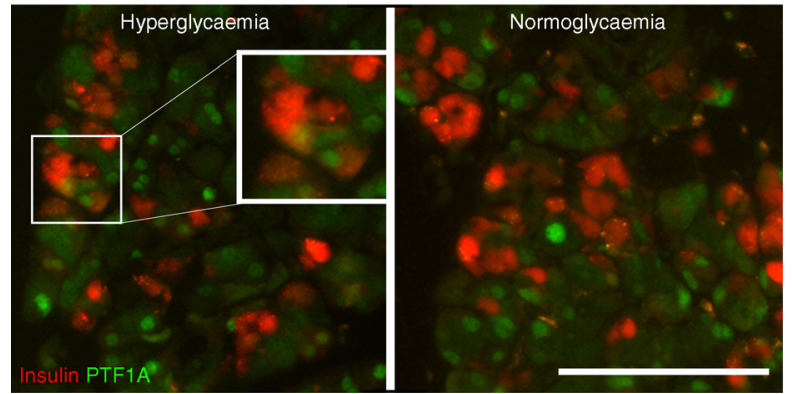
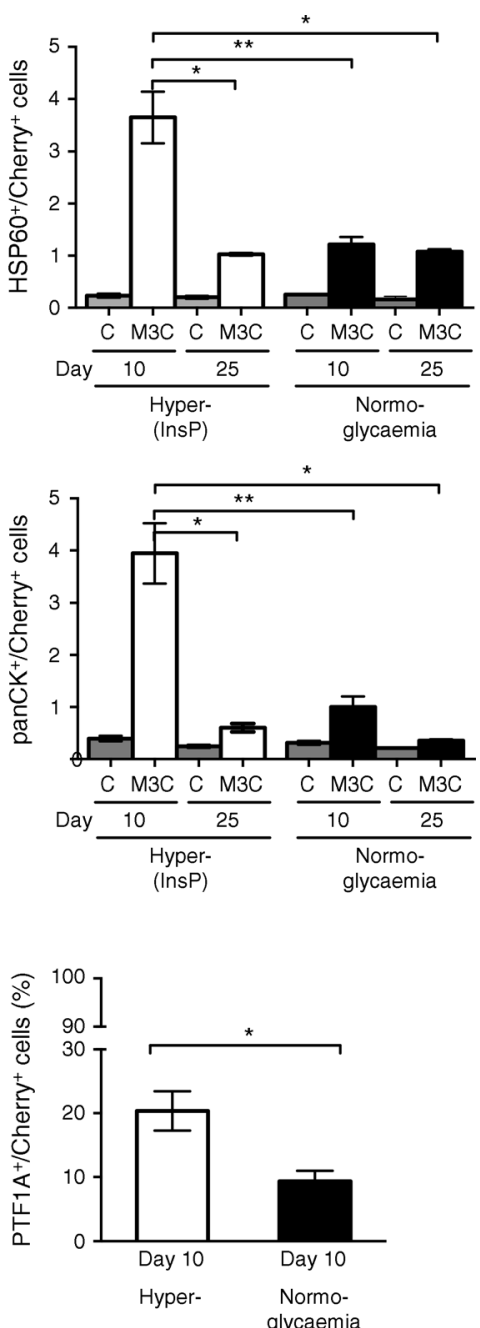

Fig. 4 In hyperglycaemia, acinar cells show transient inflammatory phenotypic changes and the acinar cell programme does not adequately shut off. (a) Tubular formation: on day 10 with hyperglycaemia, tubular structures with HSP60 expression were abundantly present in the exocrine pancreas. Quantifications of $\mathrm{HSP} 60^{+}$: Cherry $^{+}$cells yielded significantly higher ratios in hyperglycaemic $(n=5)$ compared with normoglycaemic M3Cherry-injected animals $(n=4)$ on day $10(p=0.008)$, which were gone by day 25. (b) Similar to HSP60, panCK was expressed in tubular structures of the exocrine pancreas in hyperglycaemic M3Cherry-injected

This finding indicates that primarily M2-polarised macrophages were depleted, and that their eradication exacerbated the inflammatory milieu. Confirming efficient monocyte/ macrophage depletion, the relative percentage of $\mathrm{CD}_{11} \mathrm{~b}^{+} / \mathrm{Gr} 1^{-/ \text {low }}$ monocyte relative to $\mathrm{CD} 11 \mathrm{~b}^{+} / \mathrm{Gr} 1^{\text {high }}$ granulocytes was significantly lower in the peripheral blood in macrophage-ablated animals (ESM Fig. 5). However, macrophage-depleted animals did not display increased reprogramming efficiency compared with controls (insulin ${ }^{+} /$Cherry $^{+}$cells, CD11b-DTR: $69 \pm 5 \%$ [SD], WT: $84 \pm 5 \%$ [SD]; Fig. 5c). We conclude that at least under normoglycaemia, macrophages are not directly detrimental to reprogramming and might even engage in dampening tissue inflammation. animals on day 10 , with again significantly higher ratios of panCK ${ }^{+}$Cherry $^{+}$in hyperglycaemic $(n=5)$ compared with normoglycaemic animals $(n=4)$ on days $10(p=0.008)$ and 25 $(p=0.03)$. (c) Acinar downregulation: PTF1A as a marker for acinar cell differentiation was not readily downregulated in the hyperglycaemic (insert) compared with the normoglycaemic condition ( $p<0.05, t$ test) on day 10. Immunohistochemistry images: insulin, red; HSP60/panCK/ PTF1A, green; DAPI, blue. C, Cherry; M3C, M3Cherry. ${ }^{*} p<0.05$, ${ }^{* *} p<0.01$. Scale bars, (a) and (b) $50 \mu \mathrm{m}$, (c) $130 \mu \mathrm{m}$

To better understand the role of macrophages, we assessed their spatial relationship with reprogrammed cells and found that on day 25 a substantial number of macrophages had engulfed Cherry ${ }^{+}$insulin ${ }^{-}$cells, which was most pronounced in the hyperglycaemic group (macrophages with Cherry $^{+}$insulin $^{-} 36.2 \pm 5.1 \%$ [hyper, $n=4$ ] vs $19.7 \pm 4.8 \%$ [normo, $n=4$ ]; insert Fig. 5d). The numbers of engulfed Cherry $^{+}$insulin $^{+}$cells in macrophages were generally lower (Fig. 5d). TUNEL staining to assess whether reprogrammed cells had undergone apoptosis prior to engulfment by macrophages showed that occurrence of apoptotic Cherry ${ }^{+}$cells was rare in both hyperglycaemic and normoglycaemic conditions, yet slightly higher in hyperglycaemic animals on day 10 $(p=0.03$; not shown). Thus, low apoptosis rate and at the 
Table 1 Gene set enrichment analysis

\begin{tabular}{lllll}
\hline $\begin{array}{l}\text { Gene ontology term or } \\
\text { pathway database }\end{array}$ & Term & Count & NES & FDR $q$-value \\
\hline GO, biological process & Leukocyte activation & 47 & -1.54 & 0.105 \\
KEGG & Haematopoietic cell lineage & 65 & -1.42 & 0.175 \\
KEGG & JAK-STAT signalling pathway & 130 & -1.44 & 0.217 \\
KEGG & TGF $\beta$ signalling pathway & 78 & -1.39 & 0.239 \\
KEGG & Antigen processing/presentation & 41 & -1.36 & 0.212 \\
GO, biological process & Positive regulation of T cell activation & 17 & -1.36 & 0.248 \\
KEGG & Fc epsilon RI signalling pathway & 72 & -1.33 & 0.206 \\
KEGG & Fc gamma R-mediated phagocytosis & 78 & -1.33 & 0.195 \\
KEGG & Cytokine-cytokine receptor interaction & 207 & -1.32 & 0.183 \\
KEGG & RIG-I-like receptor signalling pathway & 53 & -1.32 & 0.189 \\
KEGG & B cell receptor signalling pathway & 64 & -1.31 & 0.190 \\
KEGG & Cytotoxicity mediated by natural killer cells & 85 & -1.31 & 0.183 \\
KEGG & Apoptosis & 68 & -1.29 & 0.211 \\
\hline
\end{tabular}

The 13/25 enriched gene sets in hyperglycaemia connected with inflammation or cell death are shown (FDR $<25 \%$, ordered by descending normalised enrichment score)

GO, Gene Ontology; JAK, Janus kinase; NES, normalised enrichment score; RIG-I, retinoic acid-inducible gene 1; STAT, signal transducer and activator of transcription same time high numbers of engulfed Cherry ${ }^{+}$cells in macrophages suggests that either apoptosis is not a prerequisite for macrophage engulfment or that apoptosis is difficult to capture. Thus, in the presence of hyperglycaemia it appears that a high number of macrophages are recruited by day 10 due to the presence of incompletely reprogrammed cells, some of which are found to be engulfed by the macrophages at day 25 .

\section{Discussion}

In this study, we found that hyperglycaemia negatively affects in vivo exocrine to insulin ${ }^{+}$cell reprogramming in terms of their number, differentiation and function. Studies performed in a normoglycaemic environment yielded more than twice as many reprogrammed cells compared with hyperglycaemia. Our results stand in contrast with other studies, which have indicated that hyperglycaemia promotes reprogramming of non-beta to beta cells in a cytokine-treatment model [10] and a partial duct ligation (PDL) model [36]. Our PDL studies, however, failed to produce a similar facilitating result [37]. The mechanisms for these differing observations require further studies.

When Cherry ${ }^{+}$cells were evaluated 10 days after injection of the M3Cherry virus, they had a considerably smaller diameter (about 30\%) than those injected with the control virus. This result can be taken as evidence for a change in cell phenotype towards a smaller cell such as a beta cell because acinar cells are larger than beta cells. Of note, the reduction in cell size was essentially the same for both the normoglycaemic and hyperglycaemic conditions, which suggests that initiation of the reprogramming cascade is taking place notwithstanding hyperglycaemia. However, size reduction of reprogrammed cells does not presume stabilisation towards a fully reprogrammed phenotype [33].

The effects of hyperglycaemia became more evident when endocrine and beta cell commitment were evaluated, respectively. With normoglycaemia, virtually all Cherry ${ }^{+}$cells expressed ChgA, as an indicator for their endocrine differentiation, while in the hyperglycaemic state about $10 \%$ of the Cherry $^{+}$cell were ChgA-negative. Hyperglycaemia was also associated with a reduction of cells stained for both insulin and Cherry. Additional evidence for the adverse effects of hyperglycaemia was that $\mathrm{Cherry}^{+}$cells lacked prompt downregulation of the acinar marker PTF1A in hyperglycaemia compared with normoglycaemia. Our earlier study using this reprogramming model found that downregulation of acinar factors preceded adoption of an endocrine and beta cell phenotype [34]. Our estimation of the total number of Cherry ${ }^{+}$ cells suggests that they are similar in normoglycaemic and hyperglycaemic conditions (normo $263.5 \pm 60 \times 10^{3}$, hyper $222.7 \pm 46 \times 10^{3}$, both $\left.n=5, p=0.4\right)$. Differential adenoviral infection therefore is unlikely to account for our observations.

The impaired differentiation towards the beta cell lineage was also reflected by reduced function, which was seen when reprogrammed cells were challenged with hyperglycaemia produced by graft removal early (10 days), as opposed to later (25 day) removal after reprogramming. We found that in the normoglycaemic state the number of induced cells increased between days 10 and 25; therefore, we postulate that the number of induced cells by day 10 is only marginally sufficient to sustain normoglycaemia. 

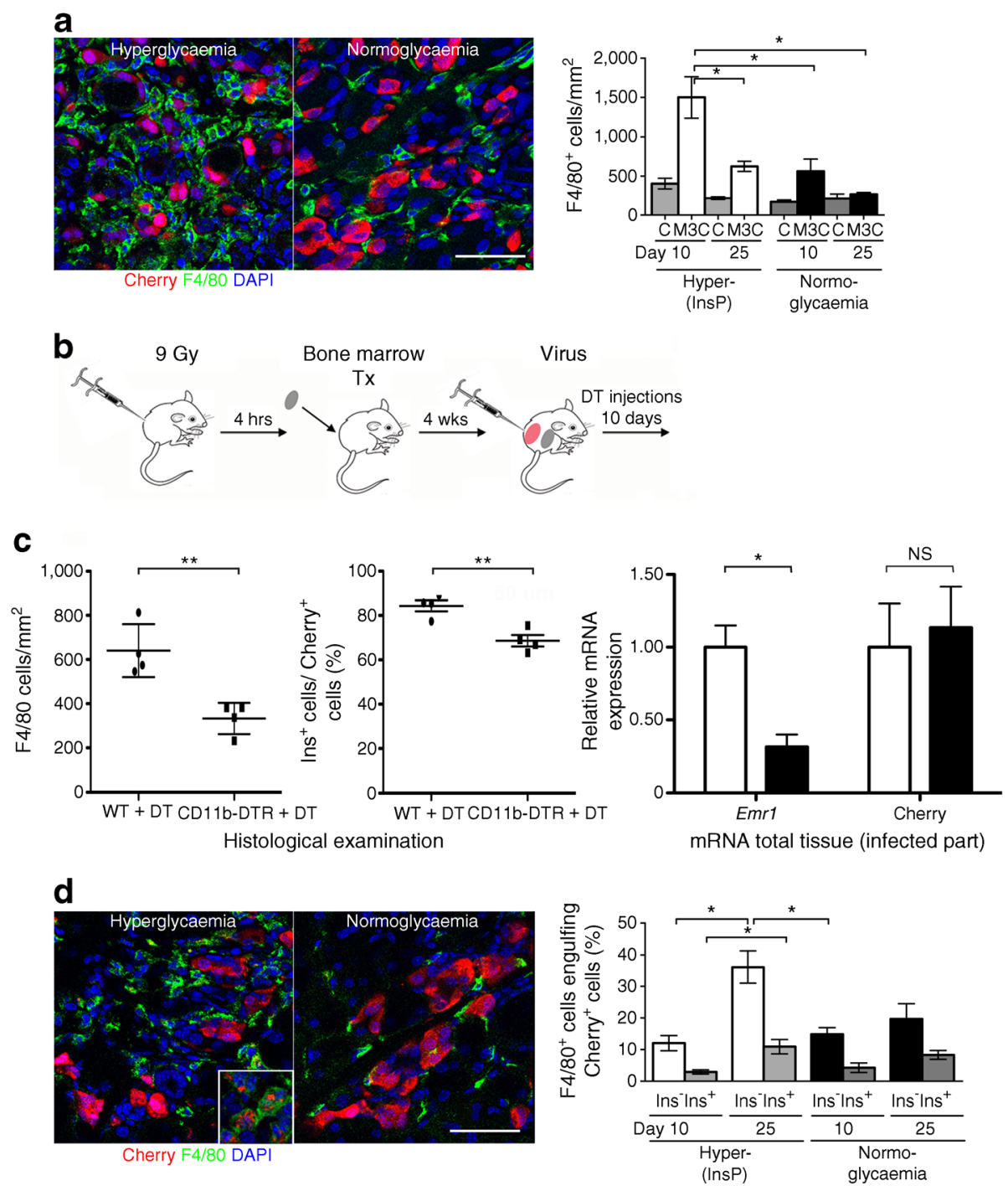

Fig. 5 Reprogramming attracts macrophages into the tissue that do not directly harm the reprogramming process but clear infected cells. (a) Macrophage accumulation: macrophages as assessed by F4/80 staining were found predominantly in hyperglycaemic as opposed to normoglycaemic animals on day $10(n=4-5)$. (b) To test whether macrophage depletion increases reprogramming efficiency, we established a model of macrophage depletion in $\mathrm{Rag}^{-/}$mice by whole body irradiation, followed by bone marrow Tx of CD11b-DTR or WT donors and DT injection for 10 days for ablation of CD11b-DTR cells. (c) On day 10, WT transplanted mice $(n=4)$ had significantly higher macrophage infiltration $\left(640 \pm 120\right.$ vs $333 \pm 71$ macrophages $\left./ \mathrm{mm}^{2}\right)$ than CD11b-DTR transplanted mice $(n=4)$ treated with DT ( 2 sections/mouse [4 per group]

Major phenotypic changes of the acinar cell compartment consisting of tubular complexes at the site of injection in the pancreatic tail were found at day 10 after M3Cherry injection in the presence of hyperglycaemia but not with normoglycaemia. Transient tubular complex formation has been described with pancreatic duct ligation [37] and cerulein-induced pancreatitis [38] and is thought to originate from acinar cells [35], while beta cells have been excluded as their source of origin [39]. However, the recovery of normal quantified). mRNA isolated from infected pancreas confirmed reduced presence of macrophages (Emrl), whereas the adenoviral Cherry transgene expression was similar. White bars, WT + DT; black bars, CD11bDTR + DT. Macrophage depletion, however, resulted in decreased rather than increased reprogramming efficiency (insulin ${ }^{+} /$Cherry $^{+}$cells WT: 84 $\pm 5 \%$, CD11b-DTR: $69 \pm 5 \%$ ). (d) On day 25, many F4/80+ cells had engulfed Cherry ${ }^{+}$cells (insert). Quantification yielded significant increases of engulfed Cherry ${ }^{+}$insulin ${ }^{-}$cells $(p=0.016)$ and Cherry ${ }^{+}$insulin ${ }^{+}$ cells from day 10 to $25(p=0.016)$ in the hyperglycaemic condition $(n=4-5)$. Means \pm SEM (except $\mathbf{c} \pm \mathrm{SD}$ ) are shown. Scale bar, $50 \mu \mathrm{m}$; F4/80, green; Cherry, red; DAPI, blue; C, Cherry; M3C, M3Cherry. $* p<0.05, * * p<0.01$

exocrine morphology is rapid (within 7 days) in ceruleininduced pancreatitis [38]. It is possible that hyperglycaemia prolongs the inflammatory changes of the exocrine pancreas elicited by the M3Cherry injection. In support of this theory, hyperglycaemia has been linked to impaired exocrine regeneration [40, 41] and impaired wound healing [42]. We found high expression of HSP60 in tubular complexes. HSP60 is a mitochondrial chaperone that promotes refolding of redoxmodified proteins [43] and has been shown to be induced in 
response to hyperglycaemia and oxidative stress in vitro (human HeLa [44] and INSE1 cells [45]).

Microarray analyses of Cherry ${ }^{+}$cells obtained from hyperglycaemic animals revealed a strong inflammatory and apoptotic pattern. Macrophages were abundant in areas of reprogramming in hyperglycaemic animals, located next to tubular complexes. The close spatial and temporal relationship between tubules and macrophages suggests a mutual interaction; perhaps secretion of biologically active factors by macrophages leads to tubular complex formation as has been postulated [46]. Mechanistically, the macrophage-secreted proinflammatory cytokines TNF and RANTES (regulated on activation, normal $\mathrm{T}$ cell expressed and secreted) have been identified to activate the nuclear factor (NF)-Kb pathway in acinar cells and by induction of its target gene MMP-9 leading to tubular complex formation [47].

If macrophages were directly deleterious to reprogramming success, reducing their number during or after viral delivery of transcription factors might have given rise to more reprogrammed cells. Thus, macrophage depletion was performed in normoglycaemic mice using CD11b-DTR. Bone marrow Tx was necessary as DT treatment of nontransplanted Rag;CD11b-DTR mice caused severe toxicity. For animal welfare reasons, such a depletion experiment could only be performed in normoglycaemic animals. The monocyte/ macrophage lineage was consistently depleted by approximately $50 \%$. We found that reduction of macrophages to this degree did not increase reprogramming efficiency. However, the only partial depletion of macrophages in our model could obscure some of their functions in reprogramming. Given that we mainly depleted M2-polarised, alternatively activated macrophages, with increased total levels of the proinflammatory cytokine $\mathrm{TNF} \alpha$, this indicates that early macrophage infiltration might serve a role in curbing inflammation. Another reason for the expanded macrophage presence in hyperglycaemic animals could also be due to increased phagocytic demand to clear the pancreas of only partially reprogrammed cells, which might be abnormal, dying or dead. Accordingly, Cherry ${ }^{+}$insulin ${ }^{-}$cells were found to be engulfed by macrophages, most prominently with hyperglycaemia on day 25 .

In summary, our studies found that hyperglycaemia impairs in vivo reprogramming of exocrine to insulin ${ }^{+}$cells. Our model proposes that hyperglycaemia causes less efficient cell reprogramming, which leads to less efficient secretory function and even cell death. In the normoglycaemic state, there must also be production of some defective cells that are cleared by macrophages, but the situation with hyperglycaemia seems much worse. The suggested sequence of events in reprogramming with hyperglycaemia is as follows: (1) less downregulation of acinar factors and less activation of the beta cell programme resulting in poorly differentiated cells that are vulnerable and dysfunctional; (2) the presence of such partially reprogrammed defective cells, probably leading to more macrophage infiltration into areas of reprogramming; and (3) remodelling of the exocrine tissue with tubular complex formation, most probably through cytokine secretion of macrophages. Macrophages may also play an important role in the clearance of damaged and dead cells. Gaining in-depth knowledge about factors influencing reprogramming success is important for a comprehensive understanding of reprogramming mechanisms and their potential for future clinical application.

Acknowledgements We thank J. Hollister-Lock (Joslin Diabetes Center, Harvard University, Boston, MA) and J. Brown (Department of Stem Cell and Regenerative Biology, Harvard University, Cambridge, MA) for technical assistance, B. Saez (Massachusetts General Hospital, Boston, MA, USA) for support for the bone marrow transplantation experiment and Boston Children's Hospital core facility for an Illumina array.

Contribution statement $\mathrm{CCW}, \mathrm{WL}, \mathrm{AZ}, \mathrm{TY}, \mathrm{JL}, \mathrm{AJ}, \mathrm{YMB}, \mathrm{GCW}$ and QZ provided substantial contributions to experimental design. CCW, WL, AZ, MSA, YZ, TY, MW, JL, AJ and YMB provided substantial contributions to experimental execution. CCW, WL, AZ, MSA, YZ, MW, SBW, GCW and QZ carried out data analyses. CCW, WL, AZ, MSA, YZ, TY, MW, JL, AJ, YMB, SBW, GCW and QZ were involved in figure preparation, manuscript writing and editing. $\mathrm{CCW}, \mathrm{WL}, \mathrm{AZ}$, MSA, YZ, TY, MW, JL, AJ, YMB, SBW, GCW and QZ approved the version to be published. QZ is the guarantor of this work.

Funding This study was supported by grants from the JDRF and the Hahnemann Hospital Foundation (GCW), and the National Institutes of Health NIH R01 DK 066056 and DK 093909 (SBW), P30 DK036836 (Joslin Diabetes Research Center and its Advanced Microscopy Core), as well as the Diabetes Research and Wellness Foundation. CCW and AZ are supported by postdoctoral fellowships from the Swiss Science Foundation and the Swiss Foundation for Grants in Biology and Medicine. WL is supported by a postdoctoral fellowship from the Juvenile Diabetes Research Foundation. QZ is supported by grants from The National Institute of Diabetes and Digestive and Kidney Diseases (NIDDK) and Harvard Stem Cell Institute (HSCI).

Duality of interest The authors declare that there is no duality of interest associated with this manuscript.

\section{References}

1. Dor Y, Brown J, Martinez OI, Melton DA (2004) Adult pancreatic beta-cells are formed by self-duplication rather than stem-cell differentiation. Nature 429:41-46

2. Nir T, Melton DA, Dor Y (2007) Recovery from diabetes in mice by beta cell regeneration. J Clin Invest 117:2553-2561

3. Teta M, Long SY, Wartschow LM, Rankin MM, Kushner JA (2005) Very slow turnover of beta-cells in aged adult mice. Diabetes 54:2557-2567

4. Bonner-Weir S, Baxter LA, Schuppin GT, Smith FE (1993) A second pathway for regeneration of adult exocrine and endocrine pancreas. A possible recapitulation of embryonic development. Diabetes 42:1715-1720

5. Bonner-Weir S, Toschi E, Inada A et al (2004) The pancreatic ductal epithelium serves as a potential pool of progenitor cells. Pediatr Diabetes 5(Suppl 2):S16-S22 
6. Inada A, Nienaber C, Katsuta H et al (2008) Carbonic anhydrase IIpositive pancreatic cells are progenitors for both endocrine and exocrine pancreas after birth. Proc Natl Acad Sci U S A 105: 19915-19919

7. Rosenberg L (1998) Induction of islet cell neogenesis in the adult pancreas: the partial duct obstruction model. Microsc Res Tech 43: 337-346

8. Rezania A, Bruin JE, Arora P et al (2014) Reversal of diabetes with insulin-producing cells derived in vitro from human pluripotent stem cells. Nat Biotechnol 32:1121-1133

9. Pagliuca FW, Millman JR, Gurtler M et al (2014) Generation of functional human pancreatic beta cells in vitro. Cell 159:428-439

10. Baeyens L, Lemper M, Leuckx G et al (2014) Transient cytokine treatment induces acinar cell reprogramming and regenerates functional beta cell mass in diabetic mice. Nat Biotechnol 32:76-83

11. Chera S, Baronnier D, Ghila L et al (2014) Diabetes recovery by age-dependent conversion of pancreatic delta-cells into insulin producers. Nature 514:503-507

12. Collombat $\mathrm{P}, \mathrm{Xu} X$, Ravassard $\mathrm{P}$ et al (2009) The ectopic expression of Pax4 in the mouse pancreas converts progenitor cells into alpha and subsequently beta cells. Cell 138:449-462

13. Ferber S, Halkin A, Cohen H et al (2000) Pancreatic and duodenal homeobox gene 1 induces expression of insulin genes in liver and ameliorates streptozotocin-induced hyperglycemia. Nat Med 6:568-572

14. Li W, Cavelti-Weder C, Zhang Y et al (2014) Long-term persistence and development of induced pancreatic beta cells generated by lineage conversion of acinar cells. Nat Biotechnol 32:1223-1230

15. Talchai C, Xuan S, Lin HV, Sussel L, Accili D (2012) Pancreatic beta cell dedifferentiation as a mechanism of diabetic beta cell failure. Cell 150:1223-1234

16. Thorel F, Nepote V, Avril I et al (2010) Conversion of adult pancreatic alpha-cells to beta-cells after extreme beta-cell loss. Nature 464:1149-1154

17. Zhou Q, Brown J, Kanarek A, Rajagopal J, Melton DA (2008) In vivo reprogramming of adult pancreatic exocrine cells to betacells. Nature 455:627-632

18. Talchai C, Xuan S, Kitamura T, DePinho RA, Accili D (2012) Generation of functional insulin-producing cells in the gut by Foxol ablation. Nat Genet 44:406-412

19. Weir GC, Bonner-Weir S (2013) Islet beta cell mass in diabetes and how it relates to function, birth, and death. Ann N Y Acad Sci 1281: 92-105

20. Porat S, Weinberg-Corem N, Tornovsky-Babaey S et al (2011) Control of pancreatic beta cell regeneration by glucose metabolism. Cell Metab 13:440-449

21. Terauchi Y, Takamoto I, Kubota N et al (2007) Glucokinase and IRS-2 are required for compensatory beta cell hyperplasia in response to high-fat diet-induced insulin resistance. J Clin Invest 117:246-257

22. Leahy JL, Bonner-Weir S, Weir GC (1992) Beta-cell dysfunction induced by chronic hyperglycemia. Current ideas on mechanism of impaired glucose-induced insulin secretion. Diabetes Care 15:442455

23. Leahy JL, Cooper HE, Deal DA, Weir GC (1986) Chronic hyperglycemia is associated with impaired glucose influence on insulin secretion. A study in normal rats using chronic in vivo glucose infusions. J Clin Invest 77:908-915

24. Leahy JL, Weir GC (1988) Evolution of abnormal insulin secretory responses during 48-h in vivo hyperglycemia. Diabetes 37:217-222

25. Weir GC, Clore ET, Zmachinski CJ, Bonner-Weir S (1981) Islet secretion in a new experimental model for non-insulin-dependent diabetes. Diabetes 30:590-595

26. Brunzell JD, Robertson RP, Lerner RL et al (1976) Relationships between fasting plasma glucose levels and insulin secretion during intravenous glucose tolerance tests. J Clin Endocrinol Metab 42: $222-229$
27. Ward WK, Bolgiano DC, McKnight B, Halter JB, Porte D Jr (1984) Diminished B cell secretory capacity in patients with noninsulindependent diabetes mellitus. J Clin Invest 74:1318-1328

28. Donath MY, Gross DJ, Cerasi E, Kaiser N (1999) Hyperglycemiainduced beta-cell apoptosis in pancreatic islets of Psammomys obesus during development of diabetes. Diabetes 48:738-744

29. Maedler K, Spinas GA, Lehmann R et al (2001) Glucose induces beta-cell apoptosis via upregulation of the Fas receptor in human islets. Diabetes 50:1683-1690

30. Cavelti-Weder C, Li W, Weir GC, Zhou Q (2014) Direct lineage conversion of pancreatic exocrine to endocrine Beta cells in vivo with defined factors. Methods Mol Biol 1150:247-262

31. Gotoh M, Maki T, Satomi S et al (1987) Reproducible high yield of rat islets by stationary in vitro digestion following pancreatic ductal or portal venous collagenase injection. Transplantation 43:725-730

32. Davalli AM, Ogawa Y, Scaglia L et al (1995) Function, mass, and replication of porcine and rat islets transplanted into diabetic nude mice. Diabetes 44:104-111

33. Smith ZD, Nachman I, Regev A, Meissner A (2010) Dynamic single-cell imaging of direct reprogramming reveals an early specifying event. Nat Biotechnol 28:521-526

34. Li W, Nakanishi M, Zumsteg A et al (2014) In vivo reprogramming of pancreatic acinar cells to three islet endocrine subtypes. Elife 3:e01846

35. Strobel O, Dor Y, Alsina J et al (2007) In vivo lineage tracing defines the role of acinar-to-ductal transdifferentiation in inflammatory ductal metaplasia. Gastroenterology 133:1999-2009

36. Pan FC, Bankaitis ED, Boyer D et al (2013) Spatiotemporal patterns of multipotentiality in Ptfla-expressing cells during pancreas organogenesis and injury-induced facultative restoration. Development 140:751-764

37. Cavelti-Weder C, Shtessel M, Reuss JE et al (2013) Pancreatic duct ligation after almost complete beta-cell loss: exocrine regeneration but no evidence of beta-cell regeneration. Endocrinology 154: 4493-4502

38. Jensen JN, Cameron E, Garay MV, Starkey TW, Gianani R, Jensen $\mathrm{J}$ (2005) Recapitulation of elements of embryonic development in adult mouse pancreatic regeneration. Gastroenterology 128:728741

39. Strobel O, Dor Y, Stirman A et al (2007) Beta cell transdifferentiation does not contribute to preneoplastic/metaplastic ductal lesions of the pancreas by genetic lineage tracing in vivo. Proc Natl Acad Sci U S A 104:4419-4424

40. Yamamoto M, Jia DM, Fukumitsu K, Otsuki M (2003) Treatment for hyperglycemia promotes pancreatic regeneration in rats without CCK-1 receptor gene expression. Pancreas 26:368-374

41. Calderari S, Chougnet C, Clemessy M, Kempf H, Corvol P, Larger E (2012) Angiopoietin 2 alters pancreatic vascularization in diabetic conditions. PLoS One 7:e29438

42. Velander P, Theopold C, Hirsch T et al (2008) Impaired wound healing in an acute diabetic pig model and the effects of local hyperglycemia. Wound Repair Regen 16:288-293

43. Ostermann J, Horwich AL, Neupert W, Hartl FU (1989) Protein folding in mitochondria requires complex formation with hsp60 and ATP hydrolysis. Nature 341:125-130

44. Hall L, Martinus RD (2013) Hyperglycaemia and oxidative stress upregulate HSP60 \& HSP70 expression in HeLa cells. Springerplus 2:431

45. Ahmed M, Muhammed SJ, Kessler B, Salehi A (2010) Mitochondrial proteome analysis reveals altered expression of voltage dependent anion channels in pancreatic beta-cells exposed to high glucose. Islets 2:283-292

46. Bockman DE (1997) Morphology of the exocrine pancreas related to pancreatitis. Microsc Res Tech 37:509-519

47. Liou GY, Doppler H, Necela B et al (2013) Macrophage-secreted cytokines drive pancreatic acinar-to-ductal metaplasia through NFKB and MMPs. J Cell Biol 202:563-577 